\title{
Effects of different drugs on the curative effect, stress responses, and the circulatory system of patients with hypertensive trigeminal neuralgia
}

\author{
Tao Lin ${ }^{1 \#}$, Jinfeng $\mathrm{Li}^{2 \#}$, Junmei Song ${ }^{3}$, Shiyin $\mathrm{Chen}^{4}, \mathrm{Hu} \mathrm{Li}^{1}$ \\ ${ }^{1}$ Department of Anesthesiology, Sichuan Academy of Medical Sciences \& Sichuan Provincial People's Hospital, University of Electronic Science \\ and Technology of China, Chengdu, China; ${ }^{2}$ Department of Anesthesiology, Chengdu Seventh People's Hospital, Chengdu, China; ${ }^{3}$ Department of \\ Cardiac Function, Sichuan Academy of Medical Sciences \& Sichuan Provincial People's Hospital, University of Electronic Science and Technology of \\ China, Chengdu, China; ${ }^{4}$ Department of Orthopedics of traditional Chinese Medicine, Sichuan Academy of Medical Sciences \& Sichuan Provincial \\ People's Hospital, University of Electronic Science and Technology of China, Chengdu, China \\ Contributions: (I) Conception and design: T Lin, J Li; (II) Administrative support: H Li, S Chen; (III) Provision of study materials or patients: All \\ authors; (IV) Collection and assembly of data: All authors; (V) Data analysis and interpretation: All authors; (VI) Manuscript writing: All authors; (VII) \\ Final approval of manuscript: All authors. \\ \#These authors contributed equally to this work. \\ Correspondence to: Shiyin Chen; Hu Li. No.32, West 2nd Section, 1st Ring Road, Chengdu 610072, China. Email: vwpnt877432@sina.cn; \\ lihusyysc1@163.com.
}

Backgroundk This research aimed to analyze the effects of stellate nerve block with different drugs on the curative effect, stress responses, and the circulatory system of patients with hypertensive trigeminal neuralgia (TN).

Methods: A retrospective analysis of 82 patients with hypertensive TN admitted to our hospital from January 2019 to January 2021 was conducted, and the patients were divided into a control group and an observation group according to different drugs. The pain visual analogue scale (VAS) scores were determined between the 2 groups before treatment and at 1,2, 3, 7, and $30 \mathrm{~d}$ after treatment. The mean arterial pressure (MAP) and heart rate (HR) were measured in the 2 groups of patients before treatment and immediately after treatment (T0), half an hour after treatment (T1), $1 \mathrm{~h}$ after treatment (T2), and $6 \mathrm{~h}$ after treatment (T3). The left ventricular wall thickness (LVWT), left ventricular end systolic volume (LVESV), left ventricular ejection fraction (LVEF), and fraction shortening (FS) were detected in the 2 groups before treatment and at T0-T2. Patient satisfaction was also scored, and the incidence of adverse reactions was assessed.

Results: The VAS scores of the 2 groups of patients decreased significantly after treatment at 1, 2, 3, 7, and 30 d. The MAP and HR indicators of the 2 groups decreased gradually at T0-T2, and gradually recovered to levels before treatment at the T3 time point. The MAP and HR indicators of the observation group were significantly lower than those of the control group at T0-T2. After treatment, the levels of LVWT, LVESV, LVEF, and FS in the observation group at the T0-T2 time points were significantly lower than those of the control group. Additionally, after treatment, the satisfaction of the observation group was significantly higher than that of the control group. The total incidence of adverse reactions in the observation group was significantly lower than that in the control group.

Conclusions: For patients with hypertensive TN, a single ropivacaine stellate nerve block can significantly relieve pain, and has little effect on heart function.

Keywords: Stellate nerve block; hypertension; trigeminal neuralgia (TN)

Submitted Apr 22, 2021. Accepted for publication Jun 08, 2021.

doi: 10.21037/apm-21-1340

View this article at: https://dx.doi.org/10.21037/apm-21-1340 


\section{Introduction}

Trigeminal neuralgia (TN) is the most common form of facial neuropathic pain. It mainly manifests as recurrent severe paroxysmal pain in the distribution area of the trigeminal nerve on one side of the face, and has a serious impact on the daily life of patients (1). Related studies have found that in hypertensive patients, the sympathetic nervous system is over-excited, and catecholamines and peripheral vascular resistance increase, which causes increases in heart rate (HR), blood pressure, and myocardial oxygen consumption. Once combined with TN, it will bring greater limitations to the clinical efficacy of treatment, and it is difficult to achieve significant therapeutic effects $(2,3)$. At present, stellate ganglion block is a common treatment for patients with TN. By blocking the stellate ganglion, pain conduction is cut off, circulation is improved, and pain is alleviated (4). However, there are still controversies regarding the choice of blocking drugs for patients with hypertension and their effects on cardiac function. This study used different drugs to treat patients with hypertensive TN in our hospital with stellate nerve block, and analyzed the effects of different drugs on patients with hypertensive TN, including their impact on stress responses and the circulatory system. We present the following article in accordance with the STROBE reporting checklist (available at http://dx.doi.org/10.21037/apm-21-1340).

\section{Methods}

\section{General information}

A retrospective analysis of 82 patients with hypertensive TN in our hospital from January 2019 to January 2021 was conducted. All patients agreed to participate in this study and signed an informed consent form. The study was conducted in accordance with the Declaration of Helsinki (as revised in 2013). This study was approved by the Sichuan Academy of Medical Sciences \& Sichuan Provincial People's Hospital, University of Electronic Science and Technology of China (No. 20181216). The patients were divided into a control group $(\mathrm{n}=41)$ and an observation group ( $\mathrm{n}=41)$ according to different drugs. The inclusion criteria were as follows: (I) all patients conformed to the diagnostic criteria for TN, including primary TN and TN caused by herpes zoster; (II) all patients conformed to the WHO diagnostic criteria for hypertension; (III) the clinical data was complete. The exclusion criteria were as follows: (I) those who were intolerant to the treatment or did not meet the indications for the treatment; (II) patients with a history of anesthetic drug allergy; (III) patients with mental or intellectual disabilities who could not cooperate with the study. There was no significant difference in general information between the 2 groups of patients $(\mathrm{P}>0.05)$, and they were comparable, as shown in Table 1.

\section{Study methods}

Patients in both groups were treated with conventional medications such as analgesics, nutrient nerves, and ipsilateral stellate ganglion block. First, a venous channel was established for the patient. The patient was placed in a supine position, with shoulder cushions, and the head was tilted later to help expose the neck and continue ECG monitoring. A portable color Doppler ultrasound (Mindray, TE7, linear array probe) was used to expose the 7 transverse processes of the affected side of the neck and the long muscles of the neck. The in-plane needle insertion method was used, and ultrasound imaging reached the surface of the cervical longus muscle at the 7 th plane of the neck. Cerebrospinal fluid (bloodless, gasless) were drawn back carefully.

The control group was injected with $1 \% 5 \mathrm{~mL}$ lidocaine (China National Pharmaceutical Group Rongsheng Pharmaceutical Company, SFDA approval number H20043676, $100 \mathrm{mg} /$ bottle) once a day on the left and right sides, with an interval of 2 hours for 5 consecutive days. The observation group was given $0.125 \% 4 \mathrm{~mL}$ ropivacaine (Chengdu Tiantaishan Pharmaceutical Co., Ltd., SFDA approval number H20052666, 75 mg/bottle) once a day for 5 consecutive days.

Echocardiogram acquisition and measurement: the acquisition method referred to the 2015 ASE/EACVI Adult Cardiac Cavity Quantitative Guidelines. Baseline data was collected on the standard apical four-chamber view, two-chamber view, and the apical four-chamber view with the right ventricle as the main heart, and 3 to 5 complete cardiac cycles were continuously recorded for storage.

\section{Observation indicators and judgment standards}

(I) Pain conditions: the visual analogue scale (VAS) (5) was used to determine pain levels 1, 2, 3, 7, and 30d after treatment. The total score was 10 points, with mild: $\leq 3$ points; moderate: 4-6 points; severe: $>6$ points.

(II) Stress response: mean arterial pressure (MAP) and 
Table 1 Comparison of general information of the 2 groups of patients

\begin{tabular}{|c|c|c|c|c|c|c|c|c|c|}
\hline Group & $\mathrm{n}$ & $\begin{array}{l}\text { Male/ } \\
\text { female }\end{array}$ & Age (year) & $\mathrm{BMI}$ & \multicolumn{2}{|c|}{ Blood pressure } & \multicolumn{3}{|c|}{ Pain area } \\
\hline $\begin{array}{l}\text { Observation } \\
\text { group }\end{array}$ & 41 & $23 / 18$ & $58.34 \pm 3.75$ & $25.32 \pm 2.11$ & $164.31 \pm 23.11$ & $95.06 \pm 12.01$ & 14 & 12 & 15 \\
\hline $\mathrm{P}$ & - & 0.823 & 0.692 & 0.670 & 0.986 & 0.982 & & 0.774 & \\
\hline
\end{tabular}

BMI, body mass index; $P$ value, probability; $\chi^{2}$-test, Chi-square test.

Table 2 Comparison of pain $(\bar{x} \pm S)$

\begin{tabular}{|c|c|c|c|c|c|c|c|}
\hline Group & $\mathrm{n}$ & \multicolumn{6}{|c|}{ VAS grade (point) } \\
\hline $\begin{array}{l}\text { Observation } \\
\text { group }\end{array}$ & 41 & $8.56 \pm 0.94$ & $7.03 \pm 0.15^{\mathrm{a}}$ & $6.93 \pm 0.05^{a}$ & $5.31 \pm 0.42^{\mathrm{abc}}$ & $4.31 \pm 0.09^{\mathrm{abcd}}$ & $2.06 \pm 0.31^{\mathrm{abcde}}$ \\
\hline $\mathrm{P}$ & - & 0.445 & 0.011 & $<0.001$ & $<0.001$ & $<0.001$ & $<0.001$ \\
\hline
\end{tabular}

Compared with before treatment, ${ }^{a} \mathrm{P}<0.05$; compared with $1 \mathrm{~d}$ after treatment, ${ }^{\mathrm{b}} \mathrm{P}<0.05$; compared with $2 \mathrm{~d}$ after treatment, ${ }^{\mathrm{c}} \mathrm{P}<0.05$; compared with $3 \mathrm{~d}$ after treatment, ${ }^{\mathrm{d}} \mathrm{P}<0.05$; compared with $7 \mathrm{~d}$ after treatment, ${ }^{\mathrm{e}} \mathrm{P}<0.05$. VAS, visual analogue scale; $\mathrm{P}$ value, probability; $t$-test, Student's $t$ test.

Heart rate (HR) of patients were measured before and after treatment in the 2 groups at immediately after treatment (T0), half an hour after treatment (T1), $1 \mathrm{~h}$ after treatment (T2), and $6 \mathrm{~h}$ after treatment (T3).

(III) Circulatory system: the levels of left ventricular wall thickness (LVWT), left ventricular end systolic volume (LVESV), left ventricular ejection fraction (LVEF), and shortened fraction (fraction shortening, FS) were detected before and after treatment in the 2 groups at T0-T2.

(IV) Treatment satisfaction: the "Therapy Satisfaction Table" made by our hospital was adopted. The survey scored a full score of 40 points, where 0 to 10 were unsatisfactory, 11 to 30 were generally satisfied, and 31 to 40 were satisfied. The Cronbach' alpha coefficient of this scale was 0.75 . Satisfaction rate $=($ satisfied + general satisfaction)/total number of cases $\times 100 \%$.

(V) The occurrence of adverse reactions: dizziness, tinnitus, nausea.

\section{Statistical analysis}

SPSS 22.0 statistical software was used to analyze the data. Measurement data were expressed as mean \pm standard deviation $(\bar{x} \pm \mathrm{s})$, and the $t / \mathrm{F}$ test was used. Count data were expressed as a percentage (\%), and the $\chi^{2}$ test was used. $\mathrm{P}<0.05$ indicated that there was a significant difference between the 2 groups.

\section{Results}

\section{Comparison of pain}

As shown in Table 2, there was no significant difference in pain before treatment between the 2 groups $(\mathrm{P}>0.05)$. The VAS scores of the 2 groups of patients at 1, 2, 3, 7, and $30 \mathrm{~d}$ after treatment were decreased compared with before treatment. The VAS score of the observation group was significantly lower than that of the control group after treatment $(\mathrm{P}<0.05)$. 


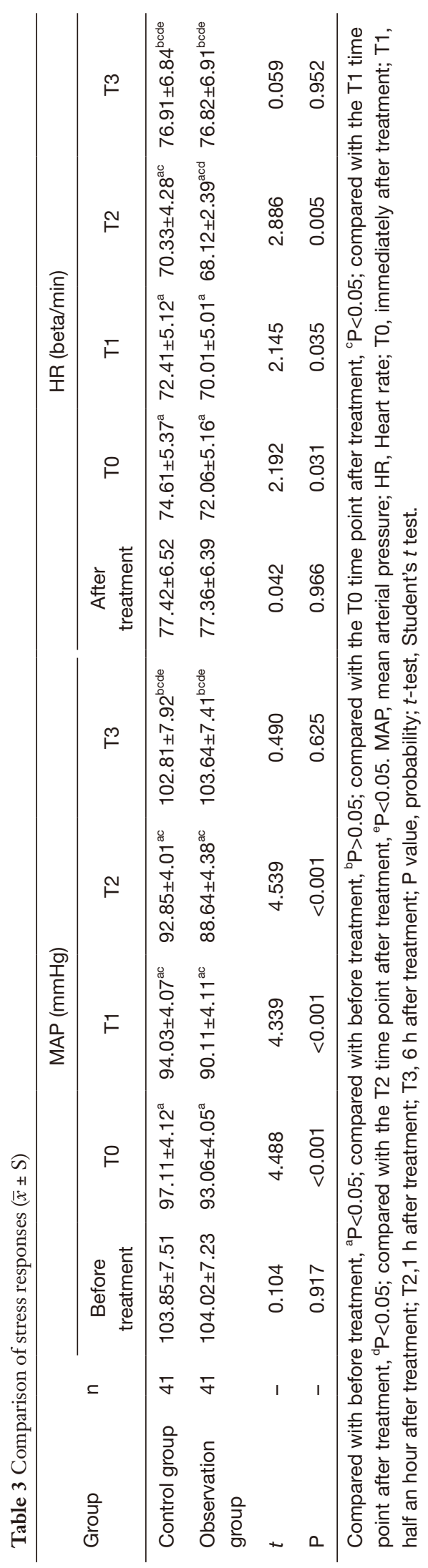

\section{Comparison of stress responses}

As shown in Table 3, the MAP and HR index levels of the 2 groups at T0-T2 gradually decreased compared with before treatment. At the T3 point, the MAP and HR index levels gradually recovered to the levels before treatment. The MAP and HR index levels of patients in the observation group at T0-T2 were significantly lower than those of the control group $(\mathrm{P}<0.05)$.

\section{Comparison of circulatory system indicators}

As shown in Table 4, after treatment, the levels of LVWT, LVESV, LVEF, and FS in the observation group at the T0$\mathrm{T} 2$ time points were significantly lower than those in the control group $(\mathrm{P}<0.05)$.

\section{Comparison of treatment satisfaction}

As shown in Table 5, the treatment satisfaction rate of the observation group (95.12\%), was significantly higher than that of the control group $(70.73 \%)(\mathrm{P}<0.05)$.

\section{Comparison of adverse reactions}

As shown in Table 6, the total incidence of complications in the observation group was significantly lower than that in the control group $(4.87 \%$ vs. $21.95 \%)(\mathrm{P}<0.05)$.

\section{Discussion}

At present, the pathogenesis of patients with hypertensive $\mathrm{TN}$ is not yet clear. However, related studies have shown that severe pain stimulation of the trigeminal nerve can cause mental tension and pain, and can induce a longterm excited state in the sympathetic nerves, which causes small arteries to contract, increases peripheral resistance, and promotes blood pressure $(6,7)$. The current clinical treatment of these patients is based on antiviral, analgesic, and neurotrophic agents. Although these have achieved certain clinical effects, they are not ideal for patients with severe pain. In addition, conventional drug treatment has a long course of treatment and significant adverse reactions, which seriously affects the quality of life of patients (8). Therefore, clinical treatment should be performed as soon as possible to control pain, so as to reduce the occurrence of neuralgia (9).

As a minimally invasive treatment method, stellate 


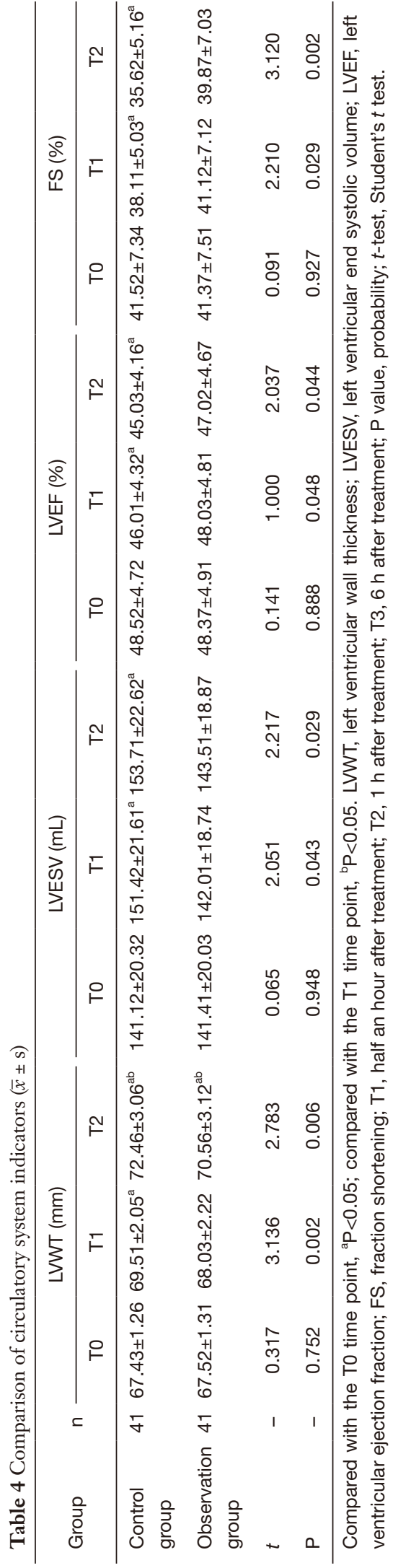

nerve block has become a research hotspot in recent years. It is widely used in daily diagnosis and treatment. It is an effective method for the treatment of neurovascular diseases such as those of the head, neck, and upper limbs. By blocking the stellate ganglion, the vicious circle of pain can be blocked and pain conduction can be cut off. It can dilate blood vessels and improve the blood supply, thereby correcting neurotrophic conditions. At the same time, it can prevent viruses from entering the nervous system by way of retrograde axons, enhance the body's defense functions, and effectively reduce the occurrence of neuralgia $(10,11)$. However, there is some controversy regarding the clinical application of nerve block drugs. Lidocaine and ropivacaine are both clinical blocking drugs. Lidocaine is an ester local anesthetic. Related studies have found that lidocaine has an inhibitory effect on the central nervous system when applied in large doses in animal experiments. Small doses have a blocking effect on the conduction of peripheral nerves. Usually, the fine fibers that conduct pain without myelin are involved first, so the analgesic effect is fast (12). Studies have shown that ropivacaine lasts for a long time, but its low fat solubility reduces the absolute effect and delays the time to reach the gross motor nerve. However, the blocking effect on $\mathrm{A} \delta$ and $\mathrm{C}$ nerve fibers is more extensive than lidocaine, and it has the advantage of separating sensory and motor block (13). There are few reports on the use of the 2 drugs on heart function in patients with hypertension. Lidocaine is an aminoacyl amide intermediate-acting local anesthetic, and has anti-arrhythmic effects. It has the advantages of high anesthesia intensity, rapid onset of action, short action time, and strong dispersion. However, it takes $2 \mathrm{~h}$ for local elimination, which easily produces rapid drug resistance. Ropivacaine is a long-acting amide local anesthetic. On the one hand, the number of punctures can be reduced to achieve a good analgesic effect. On the other hand, when used at low concentrations, it has the advantages of separation of sensory block and motor block, and has low toxicity to the cardiovascular and central nervous systems. Therefore, it has no obvious effect on myocardial contractility, blood pressure, and HR, and has a protective effect on the myocardium. A large number of previous studies have also shown that ropivacaine can minimize the entry of anesthetics into the blood, effectively alleviate myocardial ischemia and hypoxia, improve cardiac blood microcirculation, and reduce cardiotoxicity and central nervous system toxicity (13). This study showed that the levels of LVWT, LVESV, LVEF, and FS at the T0$\mathrm{T} 2$ time points after treatment with ropivacaine stellate 
Table 5 Comparison of treatment satisfaction

\begin{tabular}{|c|c|c|c|c|c|}
\hline Group & $\mathrm{n}$ & Satisfaction, n (\%) & General satisfaction, n (\%) & Dissatisfaction, n (\%) & Satisfaction rate, $\mathrm{n}(\%)$ \\
\hline Observation group & 41 & $20(48.78)$ & $9(21.95)$ & $12(29.26)$ & $29(70.73)$ \\
\hline$\chi^{2}$ & - & \multicolumn{4}{|c|}{8.613} \\
\hline$P$ & - & \multicolumn{4}{|c|}{0.003} \\
\hline
\end{tabular}

$P$ value, probability; $\chi^{2}$-test, Chi-square test.

Table 6 Comparison of adverse reactions

\begin{tabular}{|c|c|c|c|c|c|}
\hline Group & $\mathrm{n}$ & Dizziness, n (\%) & Tinnitus, n (\%) & Nausea, n (\%) & Total rate, $\mathrm{n}(\%)$ \\
\hline Observation group & 41 & $3(7.31)$ & $2(4.87)$ & $4(9.75)$ & 9 (21.95) \\
\hline$\chi^{2}$ & - & \multicolumn{4}{|c|}{5.145} \\
\hline$P$ & - & \multicolumn{4}{|c|}{0.023} \\
\hline
\end{tabular}

$P$ value, probability; $\chi^{2}$-test, Chi-square test.

nerve block were significantly lower than those of the control group. This is consistent with the previous study of Yang et al. (14) on the treatment of head and face pain with stellate ganglion block. It is believed that ropivacaine inhibits the overexcitement of sympathetic nerves, lowers blood pressure and HR, and has less impact on circulatory system function. Nielsen et al. (15) also demonstrated that ropivacaine blocks the sympathetic nerves, causing the blockage and resistance vessels to dilate. It also reduces the load on the heart, decreasing the return blood volume and blood pressure, which can inhibit the stress response to a certain extent. This is consistent with the results of this study. After treatment, the MAP and HR index levels of the observation group at T0-T2 gradually decreased compared to before treatment. At T3, the MAP and HR index levels gradually recovered to the levels before treatment. This shows that ropivacaine stellate nerve block has a significant effect on reducing stress responses.

In addition, the VAS score is used as a quantitative reference for judging pain (16). In this study, the pain relief of the observation group was significantly better than that of the control group. This suggested that the treatment of the short-term hypothalamic regulatory mechanism disorder of ropivacaine stellate ganglion is closely related to treatment. By innervating the preganglionic and postganglionic fibers of the sympathetic nerve in the surrounding area, sympathetic nerve innervation blood vessel function, muscle motor function, and pain transmission are inhibited. Furthermore, the local blood circulation has been improved, inflammation is reduced, pain is relieved, and recovery is promoted, which is beneficial to the patient's prognosis, thereby improving patient satisfaction $(17,18)$. At the same time, this study compared the adverse reactions of the 2 groups of patients. The total incidence of adverse reactions in the observation group was low, indicating that the safety of ropivacaine stellate nerve block is high. It is worth noting that stellate nerve block has high requirements for the operator. Clinicians should continuously strengthen their operational proficiency, and be proficient in treatment methods, indications, and adverse reactions to ensure the success rate of treatment.

In summary, a single ropivacaine stellate nerve block for patients with hypertensive TN can significantly relieve pain, with little effect on heart function, and has a certain degree of safety.

\section{Acknowledgments}

Funding: None.

\section{Footnote}

Reporting Checklist: The authors have completed the STROBE reporting checklist. Available at http://dx.doi. 
org/10.21037/apm-21-1340

Data Sharing Statement: Available at http://dx.doi. org/10.21037/apm-21-1340

Conflicts of Interest: All authors have completed the ICMJE uniform disclosure form (available at http://dx.doi. org/10.21037/apm-21-1340). The authors have no conflicts of interest to declare.

Ethical Statement: The authors are accountable for all aspects of the work in ensuring that questions related to the accuracy or integrity of any part of the work are appropriately investigated and resolved. The study was conducted in accordance with the Declaration of Helsinki (as revised in 2013). This study was approved by the Sichuan Academy of Medical Sciences \& Sichuan Provincial People's Hospital, University of Electronic Science and Technology of China (No. 20181216). All patients agreed to participate in this study and signed an informed consent form.

Open Access Statement: This is an Open Access article distributed in accordance with the Creative Commons Attribution-NonCommercial-NoDerivs 4.0 International License (CC BY-NC-ND 4.0), which permits the noncommercial replication and distribution of the article with the strict proviso that no changes or edits are made and the original work is properly cited (including links to both the formal publication through the relevant DOI and the license). See: https://creativecommons.org/licenses/by-nc-nd/4.0/.

\section{References}

1. Pipanmekaporn T, Saeteng S. The use of continuous thoracic paravertebral nerve block under direct vision for postoperative pain management in thoracic surgery. J Med Assoc Thai 2012;95:191-7.

2. Margus C, Correa A, Cheung W, et al. Stellate Ganglion Nerve Block by Point-of-Care Ultrasonography for Treatment of Refractory Infarction-Induced Ventricular Fibrillation. Ann Emerg Med 2020;75:257-60.

3. Gornitsky M, Elsaraj SM, Canie O, et al. Greater palatine block for V2 trigeminal neuralgia: Case report. Spec Care Dentist 2019;39:208-13.

4. Goel V, Patwardhan AM, Ibrahim M, et al. Complications associated with stellate ganglion nerve block: a systematic review. Reg Anesth Pain Med 2019. [Epub ahead of print]. doi: 10.1136/rapm-2018-100127.
5. Staker JJ, Liu D, Church R, et al. A triple-blind, placebocontrolled randomised trial of the ilioinguinal-transversus abdominis plane (I-TAP) nerve block for elective caesarean section. Anaesthesia 2018;73:594-602.

6. Zou F, Gong W, Zhang D, et al. A novel approach for performing ultrasound-guided trigeminal nerve block: Above the zygomatic arch. J Clin Anesth 2020;60:36-8.

7. Janani N, Parthasarathy S. Maxillary nerve block - a useful supplementary technique in the management of trigeminal neuralgia- a case report. SBV Journal of Basic Clinical and Applied Health Science 2019;2:43-4.

8. Piacherski V, Marochkov A. The blockade of the sciatic nerve with different doses and volumes of lidocaine using ultrasound guidance: 8AP3 6. Eur J Anaesthesiol2013;30:123.

9. Ekinci M, Ciftci B, Celik EC, et al. The Efficacy of Different Volumes on Ultrasound-Guided Type-I Pectoral Nerve Block for Postoperative Analgesia After Subpectoral Breast Augmentation: A Prospective, Randomized, Controlled Study. Aesthetic Plast Surg 2019;43:297-304.

10. Fowler JG, VanEenenaam DP Jr, Johnson KN, et al. Single midline injection for bilateral superior laryngeal nerve block. J Clin Anesth2020;66:109922.

11. Elsayed M, Alosaimy RA, Ali NY, et al. Nerve Block for Septorhinoplasty: A Retrospective Observational Study of Postoperative Complications in 24 Hours. Cureus 2020;12:e6961.

12. Al Barim B, Lemcke L, Schwake M, et al. Repetitive percutaneous radiofrequency thermocoagulation for persistent idiopathic facial pain and central neuropathic pain attributed to multiple sclerosis-a retrospective monocentric analysis. Acta Neurochir (Wien) 2020;162:2791-800.

13. Canturk M. The Canturk combination: Erector spinae plane block and femoral nerve block for postoperative analgesia after coronary artery by-pass grafting surgery. J Clin Anesth 2020;62:109700.

14. Yang DB, Wang ZM. The efficacy and safety of nerve combing for trigeminal neuralgia without neurovascular compression. Acta Neurol Belg 2019;119:439-44.

15. Nielsen TD, Moriggl B, Barckman J, et al. Randomized trial of ultrasound-guided superior cluneal nerve block. Reg Anesth Pain Med 2019. [Epub ahead of print]. doi: 10.1136/rapm-2018-100174.

16. Eckert RE, Griffin CE, Cohen ND, et al. Investigation into intraoral approach for nerve block injection at the mental foramen in the horse. Equine Veterinary Education 2019;31:328-34. 
17. Borg A, Zrinzo L. Aberrant Abducent Nerve During Microvascular Decompression for Trigeminal Neuralgiass. World Neurosurg 2020;138:454-6. Erratum in: World Neurosurg 2020;143:660.

18. Anugerah A, Nguyen K, Nader A. Technical considerations for approaches to the ultrasound-guided maxillary nerve block via the pterygopalatine fossa: a literature review. Reg Anesth Pain Med 2020;45:301-5.

(English Language Editor: C. Betlazar-Maseh)

Cite this article as: Lin T, Li J, Song J, Chen S, Li H. Effects of different drugs on the curative effect, stress responses, and the circulatory system of patients with hypertensive trigeminal neuralgia. Ann Palliat Med 2021;10(6):6833-6840. doi: 10.21037/apm-21-1340 